\title{
Hochschild cohomology of truncated quiver algebras *
}

\author{
Yunge $\mathrm{Xu}{ }^{a}$, Yang $\operatorname{Han}^{b}$ and Wenfeng Jiang ${ }^{a}$ \\ a. Faculty of Mathematics \& Computer Science, Hubei University, \\ Wuhan 430062, P.R.China E-mail: xuy@hubu.edu.cn \\ b. Academy of Mathematics and Systems science, Chinese Academy of Sciences, \\ Beijing 100080, P.R. China E-mail: hany@iss.ac.cn
}

\begin{abstract}
For a truncated quiver algebra over a field of arbitrary characteristic, its Hochschild cohomology is calculated. Moreover, it is shown that its Hochschild cohomology algebra is finitedimensional if and only if its global dimension is finite if and only if its quiver has no oriented cycles.
\end{abstract}

MSC(2000): 16E40, 16E10, 16G10

\section{Introduction}

Let $k$ be a fixed field of arbitrary characteristic and $A$ a finite-dimensional $k$-algebra. Let $A^{e}:=A^{o p} \otimes_{k} A$ be the enveloping algebra of $A$. The $n$ th Hochschild cohomology group of $A$ is $H^{n}(A):=\operatorname{Ext}_{A^{e}}^{n}(A, A)$ and the Hochschild cohomology algebra of $A$ is $H^{\bullet}(A):=\coprod_{n \geq 0} H^{n}(A)$ where the multiplication is induced by the Yoneda product for extensions [1.

Let $Q=\left(Q_{0}, Q_{1}\right)$ be a finite connected quiver where $Q_{0}$ (resp. $\left.Q_{1}\right)$ is the set of vertices (resp. arrows) in $Q$. Given a path $p$ in $Q$, we denote by $o(p)$ and $t(p)$ the origin and the terminus of $p$ respectively, and by $l(p)$ the length of $p$. For $0 \leq j \leq l(p)$, denote by ${ }^{j} p$ the subpath of $p$ with length $j$ and origin $o(p)$. A path $a_{1} \cdots a_{n}$ with $n \geq 1$ is called an oriented cycle of length $n$ if $t\left(a_{i}\right)=o\left(a_{i+1}\right)$ for $i=1, \ldots, n-1$ and $t\left(a_{n}\right)=o\left(a_{1}\right)$. An oriented cycle is called a basic cycle if it has no self intersection.

A truncated quiver algebra is an algebra $k Q / k^{n} Q$ where $n \geq 2$ and $k^{n} Q$ denotes the ideal of $k Q$ generated by all paths of length $\geq n$ (cf. [2]). It is

*Projects 10426014 and 10201004 supported by NSFC 
further called a truncated basic cycle algebra if $Q$ is a basic cycle. We refer to [3] for the theory of quivers and their representations.

Though Hochschild cohomology is theoretically computable for a concrete algebra via derived functors, actual calculation for a class of algebras is still very convenient, important and difficult. So far the Hochschild cohomology was calculated for hereditary algebras [4, 5], incidence algebras [6, 7], algebras with narrow quivers [4, 5], and so on. For the Hochschild cohomology of truncated quiver algebras partial results are known: Cibils calculated the Hochschild cohomology of radical square zero algebras [8]. In case the field $k$ is of characteristic zero, Zhang and Locateli calculated the Hochschild cohomology of truncated basic cycle algebras and truncated quiver algebras respectively 9, 10, 11. For truncated basic cycle algebras over a field $k$ of arbitrary characteristic, Bardzell, Locateli and Marcos calculated their Hochschild cohomology 12 . In this paper, we complete the calculation of the Hochschild cohomology of truncated quiver algebras over a field of arbitrary characteristic. We shall employ Locateli's strategy and do more and deeper analysis.

In 1989, Happel asked the following question: Whether for a finite dimensional algebra $A$ over an algebraically closed field $k, \operatorname{gl} . d i m . A<\infty$ if and only if the Hochschild cohomology algebra of $A$ is finite-dimensional [5]? Though Happel's question has negative answer [13], it does have some positive answers: Avramov and Iyengar's result implies that Happel's question has positive answer for commutative algebras [14. Cibils' result implies that Happel's question has positive answer for radical square zero algebras [8]. Moreover, Locateli proved that, in case the underlying field $k$ is of characteristic 0, the Hochschild cohomology algebra of a truncated quiver algebra is finite-dimensional if and only if its quiver has no oriented cycle. In this paper, we show by constructing explicitly some Hochschild cocycles that for a truncated quiver algebra over a field of arbitrary characteristic, its Hochschild cohomology algebra is finite-dimensional if and only if its global dimension is finite if and only if its quiver has no oriented cycles.

\section{Hochschild cohomology groups}

Let $A=k Q / k^{n} Q, n \geq 2$, be a truncated quiver algebra. If $S$ and $T$ are sets of paths in $Q$ we denote by $(S / / T)$ the set $\{(p, q) \in S \times T \mid o(p)=o(q)$ and $t(p)=t(q)\}$. If $S$ (resp. T) is the set of paths of length $i$ (resp. $j$ ) then we denote $(S / / T)$ by $(i / / j)$. Given a set $S, k S$ denotes the $k$-vector space with basis $S$. 
If $Q$ is a basic cycle, Bardzell, Locateli and Marcos calculated the Hochschild cohomology of $A$ :

Theorem 1. ([12, p. 1619]) Let $A=k Q / k^{n} Q$ with $Q$ a basic cycle of length $e$ and $n=m e+r, 0 \leq r \leq e-1$. Then we have

$$
\begin{gathered}
\operatorname{dim}_{k} H^{0}(A)= \begin{cases}m, & \text { if } r=0 ; \\
m+e, & \text { if } r=1 ; \\
m+1, & \text { if } r \geq 2\end{cases} \\
\operatorname{dim}_{k} H^{1}(A)= \begin{cases}m, & \text { if } r=0,1 ; \\
m+1, & \text { if } r \geq 2 .\end{cases}
\end{gathered}
$$

and for $i \geq 1$,

$\operatorname{dim}_{k} H^{2 i}(A)=\operatorname{dim}_{k} H^{2 i+1}(A)= \begin{cases}c_{n, e, i}, & \text { if chark } \nmid n \text { or } e \nmid(n i-n+1) ; \\ c_{n, e, i}+1, & \text { if chark } \mid n \text { and } e \mid(n i-n+1) .\end{cases}$

where $c_{n, e, i}:=|\{j \mid 0 \leq j \leq n-2, j \equiv \operatorname{ri}(\bmod e)\}|$.

Thanks to Theorem 1, it remains to consider the case that $Q$ is not a basic cycle. It follows from [11, Lemma 2 and Lemma 6] that for all $i \geq 0$, the $i$-th Hochschild cohomology group of $A$ is just the $i$-th cohomology group of the cochain complex

$$
\left(M^{\bullet}, d^{\bullet}\right): \quad 0 \rightarrow M^{0} \stackrel{d^{1}}{\rightarrow} M^{1} \stackrel{d^{2}}{\rightarrow} \cdots \stackrel{d^{i-1}}{\rightarrow} M^{i-1} \stackrel{d^{i}}{\rightarrow} M^{i} \stackrel{d^{i+1}}{\rightarrow} M^{i+1} \stackrel{d^{i+2}}{\rightarrow} \cdots
$$

where

$$
\begin{aligned}
& M^{2 i}=k(0 / / n i) \oplus \cdots \oplus k(n-1 / / n i), \\
& M^{2 i+1}=k(0 / / n i+1) \oplus \cdots \oplus k(n-1 / / n i+1) \text {, } \\
& d^{2 i+1}=\left[\begin{array}{ccccc}
0 & & & & \\
d_{0}^{2 i+1} & 0 & & & \\
& d_{1}^{2 i+1} & \ddots & & \\
& & \ddots & 0 & \\
& & & d_{n-2}^{2 i+1} & 0
\end{array}\right] \text {, } \\
& d^{2 i}=\left[\begin{array}{cccc}
0 & 0 & \cdots & 0 \\
\cdots & \cdots & \cdots & \cdots \\
0 & 0 & \cdots & 0 \\
d_{0}^{2 i} & 0 & \cdots & 0
\end{array}\right]
\end{aligned}
$$

with

$$
d_{j}^{2 i+1}: k(j / / n i) \rightarrow k(j+1 / / n i+1),(p, q) \mapsto \sum_{a \in Q_{1}}(a p, a q)-\sum_{a \in Q_{1}}(p a, q a)
$$


for $0 \leq j \leq n-2$ and

$$
d_{0}^{2 i}: k(0 / / n(i-1)+1) \rightarrow k(n-1 / / n i),(o(p), p) \mapsto \sum_{l(s q)=n-1}(s q, s p q) .
$$

Therefore we have

$$
\begin{gathered}
\operatorname{Ker} d^{2 i+1}=\left(\bigoplus_{j=0}^{n-2} \operatorname{Ker} d_{j}^{2 i+1}\right) \oplus k(n-1 / / n i), \quad \operatorname{Im} d^{2 i+1}=\bigoplus_{j=0}^{n-2} \operatorname{Im} d_{j}^{2 i+1}, \\
\operatorname{Ker} d^{2 i}=\operatorname{Ker} d_{0}^{2 i} \oplus \bigoplus_{j=1}^{n-1} k(j / / n(i-1)+1), \quad \operatorname{Im} d^{2 i}=\operatorname{Im} d_{0}^{2 i}, \\
H^{2 i+1}(A)=\operatorname{Ker} d_{0}^{2 i+2} \oplus \bigoplus_{j=1}^{n-1}\left(k(j / / n i+1) / \operatorname{Im} d_{j-1}^{2 i+1}\right)
\end{gathered}
$$

and

$$
H^{2 i}(A)=\left(k(n-1 / / n i) / \operatorname{Im} d_{0}^{2 i}\right) \oplus \bigoplus_{j=0}^{n-2} \operatorname{Ker} d_{j}^{2 i+1}
$$

Now we recall some notations introduced by Cibils in [2]: For $1 \leq j \leq$ $n-2$ and $(p, q) \in(j / / n i)$. We say that $(p, q)$ starts together (resp. ends together) if the first (resp. last) arrow of $p$ and $q$ coincide. And we say that $(p, q)$ starts at a source (resp. ends at a sink) if $o(p)=o(q)$ is a source (resp. if $t(p)=t(q)$ is a sink).

Suppose that $(p, q)$ starts together, so $(p, q)=\left(a p^{\prime}, a q^{\prime}\right)$ for some $a \in Q_{1}$, and moreover suppose that $(p, q)$ does not end at a sink. A +movement of $(p, q)$ is a couple $\left(p^{\prime} b, q^{\prime} b\right)$ where $b \in t(p) Q_{1}$. If $(p, q)$ does not start together, or if $(p, q)$ end at a sink, then no +movement of $(p, q)$ is defined, and we call $(p, q)$ a +extreme.

In analogous way, if $(p, q)$ ends together and $(p, q)$ does not start at a source, then we define a -movement of $(p, q)$. If $(p, q)$ does not end together, or starts at a source, we say $(p, q)$ is a -extreme.

Consider the equivalence relation on $(j / / n i)$ by declaring $(p, q)$ equivalent to $\left(p^{\prime}, q^{\prime}\right)$ if there exists a finite sequence of + or - movements transforming $(p, q)$ into $\left(p^{\prime}, q^{\prime}\right)$. We call an equivalence class a $j$-extreme if all its +extreme elements end at a sink and all its -extreme elements start at a source.

For notational convenience we denote $\{0$-extremes $\}=\emptyset$ and $\{(n-1)$ extremes $\}=(n-1 / / n i)$. One can check Locateli's proofs step by step and obtain that the following proposition and two lemmas hold for the underlying field $k$ of arbitrary characteristic. 
Proposition 1. ([11, Proposition 3]) If $Q$ is not a basic cycle, then

$$
\operatorname{dim}_{k} \operatorname{Ker} d^{2 i+1}=\sum_{j=1}^{n-1} \mid\{j \text {-extremes }\} \mid
$$

and

$$
\operatorname{dim}_{k} \operatorname{Im} d^{2 i+1}=\sum_{j=0}^{n-2}(|(j / / n i)|-\mid\{j \text {-extremes }\} \mid) .
$$

Let $x=\sum_{(p, q) \in(i / / j)} x_{(p, q)}(p, q) \in k(i / / j)$. The support of $x$ is the set $\operatorname{Supp}(x)=\left\{(p, q) \mid x_{(p, q)} \neq 0\right\}$.

Lemma 1. ([11, Lemma 7]) (Case $i=1$.) Let $(o(p), p) \in(0 / / 1)$ and $(s q, s p q) \in \operatorname{Supp}\left(d_{0}^{2 i}(o(p), p)\right)$ with $l(s q)=n-1$. Then $(s q, s p q)$ is not in the support of $d_{0}^{2 i}\left(o\left(p^{\prime}\right), p^{\prime}\right)$ for any other $p^{\prime} \neq p$.

Let $p$ and $q$ be two paths in $Q$. We denote by $p \cap q$ the set of the vertices that belong to both $p$ and $q$. Given a cycle $p=a_{1} \cdots a_{j} \cdots a_{n(i-1)+1}$ where $i \geq 2$ and $a_{j} \in Q_{1}$ for $1 \leq j \leq n(i-1)+1$, the cycle $p\left\langle a_{1} \cdots a_{j}\right\rangle:=$ $a_{j+1} \cdots a_{n(i-1)+1} a_{1} \cdots a_{j}$ is called the rotate of $p$ with origin $o\left(a_{j+1}\right)$.

Lemma 2. ([11, Lemma 8]) (Case $i \geq 2$.) Let $(o(p), p) \in(0 / / n(i-1)+$ 1) with $i \geq 2$ and $(s q, s p q) \in \operatorname{Supp}\left(d_{0}^{2 i}(o(p), p)\right)$ with $l(s q)=n-1$. Let $\left(o\left(p^{\prime}\right), p^{\prime}\right) \in(0 / / n(i-1)+1)$ and $(o(p), p) \neq\left(o\left(p^{\prime}\right), p^{\prime}\right)$. Then $(s q, s p q) \in$ $\operatorname{Supp}\left(d_{0}^{2 i}\left(o\left(p^{\prime}\right), p^{\prime}\right)\right)$ if and only if $|s q \cap p| \geq 2$ and $p^{\prime}$ is a rotate of $p$ with origin in some vertex in $s q \cap p$.

Proposition 2. If $Q$ is not a basic cycle then the map $d_{0}^{2 i}: k(0 / / n(i-$ $1)+1) \rightarrow k(n-1 / / n i)$ is injective for all $i \geq 1$.

Proof. Case $i=1$ : Let $x=\sum_{(o(p), p) \in(0 / 1)} x_{(o(p), p)}(o(p), p) \in \operatorname{Ker} d_{0}^{2}$ where $x_{(o(p), p)} \in k$. For any $(o(p), p) \in(0 / / 1)$ and $(s q, s p q) \in \operatorname{Supp}\left(d_{0}^{2}(o(p), p)\right)$, by Lemma 1 , we have that $(s q, s p q)$ is not in the support of $d_{0}^{2}\left(o\left(p^{\prime}\right), p^{\prime}\right)$ for any $p^{\prime} \neq p$. Since $d_{0}^{2}(x)=0$, we must have $x_{(o(p), p)}=0$. So $x=0$ and $d_{0}^{2}$ is injective.

Case $i \geq 2$ : Define an equivalence relation on $(0 / / n(i-1)+1)$ by saying that $p$ is equivalent to $q$ if $p$ is a rotate of $q$. Denote by $\mathcal{C}$ the set of equivalence classes. We can define a complete order in the set $(0 / / n(i-1)+1)$ as follows: First give a complete order $<^{\prime}$ to the set of classes $\mathcal{C}$. Next, for each $c \in \mathcal{C}$, choose $p=a_{1} \cdots a_{n(i-1)+1} \in c$ and order the elements of $c$ by the complete order $<^{\prime \prime}$ given by $p_{1}:=p<^{\prime \prime} p_{2}:=p\left\langle a_{1}\right\rangle<{ }^{\prime \prime} p_{3}:=p\left\langle a_{1} a_{2}\right\rangle<"$ $\cdots<p_{n(i-1)+1}:=p\left\langle a_{1} \cdots a_{n(i-1)}\right\rangle$. Then consider in $(0 / / n(i-1)+1)$ the 
lexicographic order: $(o(p), p)<(o(q), q)$ if $\bar{p}<^{\prime} \bar{q}$ or $\bar{p}=\bar{q}$ and $p<" q$, where $\bar{p}$ and $\bar{q}$ denote the equivalence classes of $p$ and $q$ respectively.

We can also give a complete order to the set $(n-1 / / n i)$ as follows: First, for any fixed equivalence class $\bar{p}=\left\{p_{1}, p_{2}, \cdots, p_{n(i-1)+1}\right\} \in \mathcal{C}$, we associate to it two subsets of $(n-1 / / n i)$ :

$$
\mathcal{B}_{\bar{p}}=\left\{\left({ }^{n-1} p_{i}, p_{i} \cdot{ }^{n-1} p_{i}\right) \in(n-1 / / n i) \mid p_{i} \in \bar{p}\right\}
$$

and

$$
\mathcal{N}_{\bar{p}}=\left\{\left(s q, s p_{i} q\right) \in(n-1 / / n i) \backslash\left(\cup_{\bar{p} \in \mathcal{C}} \mathcal{B}_{\bar{p}}\right) \mid p_{i} \in \bar{p}\right\} .
$$

Next, consider the complete order on $\mathcal{B}_{\bar{p}}$ induced by the order in $\bar{p}$. Fix a complete order in $\mathcal{N}_{\bar{p}}$ and assume that every element in $\mathcal{B}_{\bar{p}}$ is smaller than that in $\mathcal{N}_{\bar{p}}$. The order in $\mathcal{C}$ induces a complete order in $(n-1 / / n i)$ by assuming every element in $\mathcal{B}_{\bar{p}} \cup \mathcal{N}_{\bar{p}}$ is smaller than that in $\mathcal{B}_{\bar{q}} \cup \mathcal{N}_{\bar{q}}$ if $\bar{p}<\bar{q}$ in $\mathcal{C}$. Finally, given any complete order on $(n-1 / / n i) \backslash \bigcup_{c \in \mathcal{C}}\left(\mathcal{B}_{c} \cup \mathcal{N}_{c}\right)$ and require every element in $\bigcup_{c \in \mathcal{C}}\left(\mathcal{B}_{c} \cup \mathcal{N}_{c}\right)$ is smaller than that in $(n-1 / / n i) \backslash \bigcup_{c \in \mathcal{C}}\left(\mathcal{B}_{c} \cup \mathcal{N}_{c}\right)$. Thus we defined a complete order on $(n-1 / / n i)$.

Under the ordered basis of $k(0 / / n(i-1)+1)$ and $k(n-1 / / n i)$ respectively, the matrix of $d_{0}^{2 i}$ can be written in the form

$$
\left[\begin{array}{cccc}
B_{1} & & & \\
N_{1} & & & \\
& B_{2} & & \\
& N_{2} & & \\
& & \ddots & \\
& & & B_{s} \\
& & & N_{s} \\
0 & 0 & \cdots & 0
\end{array}\right]_{|(n-1 / / n i)| \times|(0 / / n(i-1)+1)|}
$$

where each submatrix $\left[\begin{array}{c}B_{j} \\ N_{j}\end{array}\right]$ corresponds to $\left.d_{0}^{2 i}\right|_{k c_{j}}$ and $\mathcal{C}=\left\{c_{1}, c_{2}, \cdots, c_{s}\right\}$.

Given $(o(p), p) \in(0 / / n(i-1)+1)$, Lemma 2 assures us that $\left({ }^{n-1} p, p\right.$. $\left.{ }^{n-1} p\right) \in \operatorname{Supp}\left(d_{0}^{2 i}\left(o\left(p^{\prime}\right), p^{\prime}\right)\right)$ if and only if $p^{\prime}$ is a rotate of $p$ and start at some vertex in $\left({ }^{n-1} p\right) \cap p$. Let $e$ be the length of the smallest cycle $w$ such that $p=w^{h}$ for some $h \geq 1$.

(i). $n=2$ and char $k \neq 2$ : It follows from $e \mid(n(i-1)+1)$ that $e \neq 2$. If $e=1$, then $p=a^{2 i-1}$ for some loop $a$ and $\bar{p}=\{p\}$. Since $Q$ is not a basic 
cycle, $B_{\bar{p}}=(2)$ and $N_{\bar{p}}$ is in the form

$$
\left[\begin{array}{c}
1 \\
1 \\
\vdots \\
1
\end{array}\right]
$$

Hence

$$
\operatorname{rank}\left[\begin{array}{c}
B_{\bar{p}} \\
N_{\bar{p}}
\end{array}\right]=1=e .
$$

If $e>2$, the rank of the matrix

$$
B_{\bar{p}}=\left[\begin{array}{ccccc}
1 & 1 & & & \\
& 1 & 1 & & \\
& & 1 & \ddots & \\
& & & \ddots & 1 \\
1 & & & & 1
\end{array}\right]_{e \times e}
$$

is

$$
\operatorname{rank} B_{\bar{p}}= \begin{cases}e, & \text { if char } k \neq 2 \\ e-1, & \text { if char } k=2 .\end{cases}
$$

Since char $k \neq 2$, we have $\operatorname{rank} B_{\bar{p}}=e$.

Each block $B_{\bar{p}}, \bar{p} \in \mathcal{C}$, is of full rank, so the map $d_{0}^{2 i}$ is injective.

(ii). $n>2$ and char $k \nmid n$ : We write $n=m e+r$, with $0 \leq r<e$. It is not difficult to see that $\left({ }^{n-1} q, q \cdot{ }^{n-1} q\right)$ in $\mathcal{B}_{\bar{p}}$ appears in $d_{0}^{2 i}(o(p), p)$ with coefficient $m+1$ if $q=p_{j}$ with $1 \leq j \leq r$, or $m$ if $q=p_{j}$ with $j>r$. Thus each matrix $B_{\bar{p}}$ is of the form

$$
B_{\bar{p}}=\left[\begin{array}{ccccc}
m+1 & m & m & \cdots & m+1 \\
m+1 & m+1 & m & \cdots & m+1 \\
m+1 & m+1 & m+1 & \cdots & m+1 \\
\vdots & \vdots & \vdots & & \vdots \\
m+1 & m+1 & m+1 & \cdots & m+1 \\
m+1 & m+1 & m+1 & \cdots & m \\
m & m+1 & m+1 & \cdots & m \\
m & m & m+1 & \cdots & m \\
\vdots & \vdots & \vdots & & \vdots \\
m & m & m & \cdots & m \\
m & m & m & \cdots & m+1
\end{array}\right]_{\text {exe }}
$$


which is a cyclic matrix, where in each column we have $r$ entries $(m+1)$ and $(e-r)$ entries $m$.

Note that if $(o(p), p) \in(0 / / n(i-1)+1)$ then one must have $n i \equiv n-1$ $(\bmod l(p))$. So $\operatorname{gcd}(n, l(p))=1$ and thus $\operatorname{gcd}(e, r)=1$. By [12, p. 1619], we have

$$
\operatorname{rank} B_{\bar{p}}= \begin{cases}e, & \text { if char } k \nmid n ; \\ e-1, & \text { if char } k \mid n .\end{cases}
$$

Since char $k \nmid n$, we have $\operatorname{rank} B_{\bar{p}}=e$.

Each block $B_{\bar{p}}, \bar{p} \in \mathcal{C}$, is of full rank, so the map $d_{0}^{2 i}$ is injective.

(iii). $n>2$ and char $k \mid n$, or $n=2$ and char $k=2$ : Since $Q$ is connected but not a basic cycle, without loss of generality, we may assume that $Q$ contains an arrow $b$ which does not appear in $w$ and $t(b)=o(p)$. Of course it is possible that $o(b)$ belongs to the vertices of $w$. Moreover, we assume $w=a_{1} a_{2} \cdots a_{e}$ and $p=w^{h}$ for some $h \geq 1$ and $n=m e+r$, with $0 \leq r<e$. Clearly, $\left(b \cdot{ }^{n-2} p, b p \cdot{ }^{n-2} p\right) \in \mathcal{N}_{\bar{p}}$ and ${ }^{n-2} p=w^{m} \cdot{ }^{r-2} p$.

(1). If $m=0$ then $n-2=r-2$, thus $n-2<e-2$. Hence

$$
\begin{aligned}
\left(b \cdot{ }^{n-2} p, b p \cdot{ }^{n-2} p\right) & =\left(b \cdot a_{1} \cdots a_{n-2}, b\left(a_{1} \cdots a_{e}\right) a_{1} \cdots a_{n-2}\right) \\
& =\left(b a_{1} \cdot a_{2} \cdots a_{n-2}, b a_{1}\left(a_{2} \cdots a_{e} a_{1}\right) a_{2} \cdots a_{n-2}\right) \\
& =\cdots \\
& =\left(b a_{1} \cdots a_{n-2}, b a_{1} \cdots a_{n-2}\left(a_{n-1} \cdots a_{e} a_{1} \cdots a_{n-2}\right)\right) .
\end{aligned}
$$

So $\left(b \cdot{ }^{n-2} p, b p \cdot{ }^{n-2} p\right)$ appears only in $d_{0}^{2 i}\left(o\left(p^{\prime}\right), p^{\prime}\right)$ with coefficient 1 for $p^{\prime}=p_{j}$ with $1 \leq j \leq n-1$, and with coefficient 0 otherwise. Thus the row corresponding to $\left(b \cdot{ }^{n-2} p, b p \cdot{ }^{n-2} p\right)$ in the matrix $N_{\bar{p}}$ is of the form

$$
(\underbrace{1,1, \cdots, 1}_{n-1} 0,0, \cdots, 0)
$$

whose row-sum is $n-1 \neq 0$. Therefore

$$
\operatorname{rank}\left[\begin{array}{c}
B_{\bar{p}} \\
N_{\bar{p}}
\end{array}\right]=e .
$$

(2). If $m \geq 1$ and $r \geq 2$ then $\left(b \cdot{ }^{n-2} p, b p \cdot{ }^{n-2} p\right)$ appears in $d_{0}^{2 i}\left(o\left(p^{\prime}\right), p^{\prime}\right)$ for $p^{\prime}=p_{j}$ with coefficient $m+1$ if $1 \leq j<r$, or $m$ if $r \leq j \leq e$. So the row of the matrix $N_{\bar{p}}$ corresponding to $\left(b \cdot{ }^{n-2} p, b p \cdot{ }^{n-2} p\right)$ is of the form

$$
(\underbrace{m+1, m+1, \cdots, m+1}_{r-1}, \underbrace{m, \cdots, m}_{e-r+1})
$$


whose row-sum is $n-1 \neq 0$. Thus

$$
\operatorname{rank}\left[\begin{array}{c}
B_{\bar{p}} \\
N_{\bar{p}}
\end{array}\right]=e .
$$

(3). If $m \geq 1$ and $r=1$ then ${ }^{n-2} p=w^{m-1} a_{1} a_{2} \cdots a_{e-1}$. So the coefficient of $\left(b \cdot{ }^{n-2} p, b p \cdot{ }^{n-2} p\right)$ in $d_{0}^{2 i}\left(o\left(p^{\prime}\right), p^{\prime}\right)$ for $p^{\prime}=p_{j}$ is $m$ if $1 \leq j \leq e$. Thus the row corresponding to $\left(b \cdot{ }^{n-2} p, b p \cdot{ }^{n-2} p\right)$ in $N_{\bar{p}}$ is of the form

$$
(\underbrace{m, m, \cdots, m}_{e})
$$

whose row-sum is also $(n-1)$ and hence nonzero. So

$$
\operatorname{rank}\left[\begin{array}{c}
B_{\bar{p}} \\
N_{\bar{p}}
\end{array}\right]=e .
$$

(4). If $m \geq 1$ and $r=0$ then ${ }^{n-2} p=w^{m-1} a_{1} a_{2} \cdots a_{e-2}$. The row corresponding to $\left(b \cdot{ }^{n-2} p, b p \cdot{ }^{n-2} p\right)$ in $N_{\bar{p}}$ is of the form

$$
(\underbrace{m, m, \cdots, m}_{e-1}, m-1)
$$

whose row-sum is $n-1 \neq 0$. Therefore

$$
\operatorname{rank}\left[\begin{array}{c}
B_{\bar{p}} \\
N_{\bar{p}}
\end{array}\right]=e
$$

Anyway, each block $\left[\begin{array}{c}B_{\bar{p}} \\ N_{\bar{p}}\end{array}\right], \bar{p} \in \mathcal{C}$, is of full rank, so the map $d_{0}^{2 i}$ is injective.

By Proposition 2, we immediately have:

Proposition 3. If $Q$ is not a basic cycle then, for $i \geq 1$, we have

$$
\operatorname{dim}_{k} \operatorname{Ker} d^{2 i}=\sum_{j=1}^{n-1}|(j / / n(i-1)+1)|
$$

and

$$
\operatorname{dim}_{k} \operatorname{Im} d^{2 i}=|(0 / / n(i-1)+1)|
$$

Now we can complete the calculation of the Hochschild cohomology of truncated quiver algebras over a field of arbitrary characteristic as follows: 
Theorem 2. Let $A=k Q / k^{n} Q$ be a truncated algebra. If $Q$ is not a basic cycle quiver then we have

$$
\begin{gathered}
H^{0}(A)=Z(A) \\
\operatorname{dim}_{k} H^{1}(A)=\operatorname{dim}_{k} Z(A)-\sum_{j=0}^{n-1}|(j / / 0)|+\sum_{j=1}^{n-1}|(j / / 1)|
\end{gathered}
$$

and for $i \geq 1$

$$
\begin{gathered}
\operatorname{dim}_{k} H^{2 i+1}(A)=\sum_{j=1}^{n-1}|(j / \mid n i+1)|-\sum_{j=0}^{n-2}(|(j / \mid n i)|-\mid\{j \text {-extremes }\} \mid) \\
\operatorname{dim}_{k} H^{2 i}(A)=\sum_{j=1}^{n-1} \mid\{j \text {-extremes }\}|-|(0 / / n(i-1)+1) \mid
\end{gathered}
$$

where $Z(A)$ denotes the center of the algebra $A$.

Proof. It is well-known that $H^{0}(A)=Z(A)$. Since $H^{0}(A)=\operatorname{Ker} d^{1}$, we have

$$
\operatorname{dim}_{k} \operatorname{Im} d^{1}=\operatorname{dim}_{k} M^{0}-\operatorname{dim}_{k} \operatorname{Ker} d^{1}=\sum_{j=0}^{n-1}|(j / / 0)|-\operatorname{dim}_{k} Z(A) .
$$

By Proposition 3, we have

$\operatorname{dim}_{k} H^{1}(A)=\operatorname{dim}_{k} \operatorname{Ker} d^{2}-\operatorname{dim}_{k} \operatorname{Im} d^{1}=\sum_{j=1}^{n-1}|(j / / 1)|-\sum_{j=0}^{n-1}|(j / / 0)|+\operatorname{dim}_{k} Z(A)$.

If $i \geq 1$ then by Proposition 1 and Proposition 3 we have

$$
\begin{aligned}
\operatorname{dim}_{k} H^{2 i+1}(A) & =\operatorname{dim}_{k} \operatorname{Ker} d^{2(i+1)}-\operatorname{dim}_{k} \operatorname{Im} d^{2 i+1} \\
& =\sum_{j=1}^{n-1}|(j / / n i+1)|-\sum_{j=0}^{n-2}(|(j / / n i)|-\mid\{j \text {-extremes }\} \mid)
\end{aligned}
$$

and

$$
\begin{aligned}
\operatorname{dim}_{k} H^{2 i}(A) & =\operatorname{dim}_{k} \operatorname{Ker} d^{2 i+1}-\operatorname{dim}_{k} \operatorname{Im} d^{2 i} \\
& =\sum_{j=1}^{n-1} \mid\{j \text {-extremes }\}|-|(0 / / n(i-1)+1) \mid
\end{aligned}
$$




\section{Hochschild cohomolgy algebras.}

The main result of this section is the following:

Theorem 3. Let $A=k Q / k^{n} Q$ be a truncated quiver algebra. Then the following conditions are equivalent:

(1) The algebra $H^{*}(A)$ is finite-dimensional;

(2) gl.dim. $A<\infty$;

(3) $Q$ has no oriented cycles.

Proof. $(3) \Rightarrow(2) \Rightarrow(1)$ : Clearly.

$(1) \Rightarrow(3)$ : If $Q$ has an oriented cycle then we take the shortest oriented cycle $w=a_{1} a_{2} \cdots a_{e}$ in $Q$. Thus the vertices of $w$ must be different from each other.

Now we show that $\left(a_{e}, a_{e}\left(a_{1} \cdots a_{e}\right)^{n r}\right) \in k(1 / / n r e+1) \backslash \operatorname{Im} d_{0}^{2 r e+1}$, thus $d_{0}^{2 r e+1}$ is not surjective, hence $H^{2 r e+1}(A) \neq 0$, for all $r \geq 0$ :

Assume on the contrary $\left(a_{e}, a_{e}\left(a_{1} \cdots a_{e}\right)^{n r}\right) \in \operatorname{Im} d_{0}^{2 r e+1}$. Then there is $\sum_{i=1}^{e} x_{i}\left(v_{i},\left(a_{i} \cdots a_{e} a_{1} \cdots a_{i-1}\right)^{n r}\right)+\sum_{j=1}^{s} y_{j}\left(u_{j}, b_{j 1} \cdots b_{j(n r e)}\right) \in k(0 / / n r e)$ with $\left\{b_{j 1}, \ldots, b_{j(n r e)}\right\} \nsubseteq\left\{a_{1}, \ldots, a_{e}\right\}$ such that $\left(a_{e}, a_{e}\left(a_{1} \cdots a_{e}\right)^{n r}\right)$

$$
\begin{aligned}
= & d_{0}^{2 r e+1}\left(\sum_{i=1}^{e} x_{i}\left(v_{i},\left(a_{i} \cdots a_{e} a_{1} \cdots a_{i-1}\right)^{n r}\right)+\sum_{j=1}^{s} y_{j}\left(u_{j}, b_{j 1} \cdots b_{j(n r e)}\right)\right) \\
= & \sum_{i=1}^{e} x_{i} \sum_{a \in Q_{1}}\left(\left(a v_{i}, a\left(a_{i} \cdots a_{e} a_{1} \cdots a_{i-1}\right)^{n r}\right)-\left(v_{i} a,\left(a_{i} \cdots a_{e} a_{1} \cdots a_{i-1}\right)^{n r} a\right)\right) \\
& +\sum_{j=1}^{s} y_{j} \sum_{a \in Q_{1}}\left(\left(a u_{j}, a b_{j 1} \cdots b_{j(n r e)}\right)-\left(u_{j} a, b_{j 1} \cdots b_{j(n r e)} a\right)\right) \\
= & \sum_{i=1}^{e} x_{i+1}\left(a_{i}, a_{i}\left(a_{i+1} \cdots a_{e} a_{1} \cdots a_{i}\right)^{n r}\right) \\
& \left.-\sum_{i=1}^{e} x_{i}\left(a_{i},\left(a_{i} \cdots a_{e} a_{1} \cdots a_{i-1}\right)^{n r}\right) a_{i}\right) \\
& +\sum_{i=1}^{e} x_{i} \sum_{a \in Q_{1} \backslash\left\{a_{i-1}\right\}}\left(a v_{i}, a\left(a_{i} \cdots a_{e} a_{1} \cdots a_{i-1}\right)^{n r}\right) \\
& -\sum_{i=1}^{e} x_{i} \sum_{a \in Q_{1} \backslash\left\{a_{i}\right\}}\left(v_{i} a,\left(a_{i} \cdots a_{e} a_{1} \cdots a_{i-1}\right)^{n r} a\right) \\
& +\sum_{j=1}^{s} y_{j} \sum_{a \in Q_{1}}\left(\left(a u_{j}, a b_{j 1} \cdots b_{j(n r e)}\right)-\left(u_{j} a, b_{j 1} \cdots b_{j(n r e)} a\right)\right)
\end{aligned}
$$

where $x_{e+1}:=x_{1}$.

Let $W$ be the $k$-subspace of $k(1 / / n r e+1)$ generated by $e$ elements $\left(a_{i}, a_{i}\left(a_{i+1} \cdots a_{e} a_{1} \cdots a_{i}\right)^{n r}\right), 1 \leq i \leq e$. Then $k(1 / / n r e+1)=W \oplus \bar{W}$ where $\bar{W}$ is the complement of $W$ in $k(1 / / n r e+1)$. According to this decomposition we have $\left(a_{e}, a_{e}\left(a_{1} \cdots a_{e}\right)^{n r}\right)=\sum_{i=1}^{e} x_{i+1}\left(a_{i}, a_{i}\left(a_{i+1} \cdots a_{e} a_{1} \cdots a_{i}\right)^{n r}\right)-$ 
$\left.\sum_{i=1}^{e} x_{i}\left(a_{i},\left(a_{i} \cdots a_{e} a_{1} \cdots a_{i-1}\right)^{n r}\right) a_{i}\right)$. Comparing the coefficients on two sides we obtain

$$
\begin{aligned}
& 1=x_{1}-x_{e} \\
& 0=x_{2}-x_{1} \\
& 0=x_{3}-x_{2} \\
& \cdots \cdots \\
& 0=x_{e}-x_{e-1} .
\end{aligned}
$$

It is a contradiction.

\section{References}

[1] Mac Lane S., Homology, Grundlehren 114, Third corrected printing, Springer-Verlag, 1975.

[2] Cibils C., Rigidity of truncated quiver algebras, Adv. Math., 1990, 79: 18-42.

[3] Auslander M., Reiten I. and Smalø S.O., Representation theory of artin algebras, Cambridge studies in advanced mathematics 36, Cambridge university press, Cambridge, 1995.

[4] Cibils C., On the Hochschild cohomology of finite-dimensional algebras, Comm. Algebra, 1988, 16: 645-649.

[5] Happel D., Hochschild cohomology of finite-dimensional algebras, Springer Lecture Notes in Math. 1404, 1989: 108-126.

[6] Cibils C., Cohomology of incidence algebras and simplicial complexes, J. Pure Appl. Algebra, 1989, 56: 221-232.

[7] Gerstenhaber M. and Schack S.P., Simplicial homology is Hochschild cohomology, J. Pure Appl. Algebra, 1983, 30: 143-156.

[8] Cibils C., Hochschild cohomology algebra of radical square zero algebras, CMS Conf. Proc. 1998, 24: 93-101.

[9] Zhang P., Hochschild cohomology of truncated algebras, Sci. China, Ser. A (in Chinese), 1994, 24: 1121-1125.

[10] Zhang P., Hochschild cohomology of truncated basic cycle, Sci. China, Ser. A, 1997, 40: $1272-1278$.

[11] Locateli A.C., Hochschild cohomology of truncated quiver algebras, Comm. Algebra, 1999, 27: 645-664.

[12] Bardzell M.J., Locateli A.C. and Marcos E.N., On the Hochschild cohomology of truncated cycle algebras, Comm. Algebra, 2000, 28: 1615-1639.

[13] Buchweitz R.O., Green E.L., Madsen D. and Solberg Ø., Finite Hochschild cohomology without finite global dimension, Math. Res. Letters (in press).

[14] Avramov L.L. and Iyengar S., Gaps in Hochschild cohomology imply smoothness for commutative algebras, Math. Res. Letters (in press). 